\title{
Les inventions de la musique au Moyen Âge, conclusions
}

Auxerre, Centre d'études médiévales, atelier du 17 au 19 juin 2009

\section{Daniel Russo}

\section{(2) OpenEdition}

\section{Journals}

Édition électronique

URL : https://journals.openedition.org/cem/11531

DOI : 10.4000/cem. 11531

ISSN : 1954-3093

Éditeur

Centre d'études médiévales Saint-Germain d'Auxerre

Édition imprimée

Pagination : 149-156

ISSN : 1623-5770

Référence électronique

Daniel Russo, «Les inventions de la musique au Moyen Âge, conclusions », Bulletin du centre d'études médiévales d'Auxerre | BUCEMA [En ligne], 14 | 2010, mis en ligne le 14 octobre 2010, consulté le 22 septembre 2022. URL : http://journals.openedition.org/cem/11531 ; DOI : https://doi.org/10.4000/ cem. 11531

Ce document a été généré automatiquement le 22 septembre 2022.

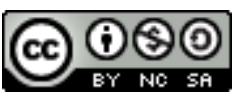

Creative Commons - Attribution - Pas d'Utilisation Commerciale - Partage dans les Mêmes Conditions 4.0 International - CC BY-NC-SA 4.0

https://creativecommons.org/licenses/by-nc-sa/4.0/ 


\title{
Les inventions de la musique au Moyen Âge, conclusions
}

Auxerre, Centre d'études médiévales, atelier du 17 au 19 juin 2009

\author{
Daniel Russo
}

1 Je commencerai par remercier les organisateurs de la rencontre, Martine Clouzot et Eduardo Aubert, pour la richesse du programme proposé à la discussion et pour l'idée première de réunir les différents intervenants autour de ces questions et de cette problématique de recherche.

2 Je soulignerai, ensuite, quatre lignes principales qui me paraissent devoir retracer l'ensemble des échanges au cours de ces journées très denses.

\section{$1^{\text {re }}$ ligne}

3 Une première direction a été ouverte pour tenter de définir et de circonscrire la musique médiévale, ou ce que nous avons nommé telle, ses usages et ses pratiques saisis entre tradition et invention, mais surtout à partir des textes savants, moins à partir des documents en langue vernaculaire - Martine Clouzot, Jean-Marie Fritz, pour les XIII ${ }^{\mathrm{e}}$ $\mathrm{XIV}^{\mathrm{e}}$ siècles, voire début $\mathrm{du} \mathrm{XV}^{\mathrm{e}}$ siècle. Du reste, il me semble utile d'ouvrir plus encore la réflexion vers les sources disponibles, en latin mais aussi en langue vulgaire, d'en esquisser une typologie par genre et par statut, et d'analyser l'emploi des mots et des familles de mots, pour mieux marquer les contours d'un champ sémantique et les contextes possibles d'utilisation. C'est ainsi que les termes rythmus, musica, cantus, et l'étude qu'en a menée Jean-Claude Schmitt, du double point de vue anthropologique et historique, dans la Tapisserie de Bayeux et la Chronique en images du roi allemand Henri VII (xIV ${ }^{\mathrm{e}}$ siècle), ont laissé voir la polysémie de la notion centrale de musique et introduit au cœur de la problématique du sujet à situer entre discontinuité et continuité, sequentia et concordia, en une tension dialectique qui apparut alors comme l'une des constantes majeures. L'analyse des lettrines figurées dans le manuscrit de Semur 1, vers l'an Mil, par Daniel Russo, a insisté, de même, sur l'idée d'une trame continue qu'on montrait dans la discontinuité du visuel à l'intérieur d'un système graphique. Dans la comparaison des 
Benedictiones bénéventaines et milanaises, Marie Formarier revenait sur la question du rythmus et mettait en valeur toute la richesse d'une formalisation aboutie, qui supportait des modes de compréhension très variés. Quelque deux siècles après, à la cathédrale Saint-Julien du Mans, dans la chapelle d'axe consacrée à Marie, la Vierge Mère de Dieu, toute une série de petits compartiments où étaient inscrits des anges musiciens faisaient ressortir, ainsi qu'Helen Deeming les présentait, une aussi grande formalisation développée à l'intersection des pratiques vocales et des techniques de la peinture murale, et thématisée à travers la liturgie qu'elle devait, sans doute, accompagner. Au XIV siècle, il est vrai, la formalisation servait de base à toute une argumentation théologique, médiatisée par la liturgie, et transformait le chant comme l'image en des arguments pour la prédication de l'Église et en des figures de rhétorique appliquées à tous les signes, vocaux et graphiques, c'est-à-dire textuels et visuels ${ }^{1}$.

4 Ces pratiques, ces usages, de plus en plus étendus et complexifiés, provenaient de toute une suite de mutations, observées par Guillaume Gross, autour de la polyphonie chantée à Notre-Dame de Paris dès les années 1200, et donnant lieu à l'organum, synthèse aboutie dans l'ordre du verbal et du visuel, pour laquelle jouait à plein l'équivalence assumée entre la rhétorique et la musique. Discutant la notion scolaire de l'habitus, formée dans les écoles urbaines et expérimentée dans l'abbaye de Saint-Victor à Paris, Guillaume Gross marquait l'importance de ce point tournant aussi bien dans les arts et leurs pratiques, que dans la mise en ordre du social: car l'organum est tout à la fois le résultat d'un ensemble opératoire de techniques intellectuelles, un appareil mental et, plus encore, une vision de la société chrétienne, ainsi qu'Edgar de Bruyne, Erwin Panofsky et Pierre Bourdieu l'avaient aussi établi ${ }^{2}$. Au cours du XIII ${ }^{\mathrm{e}}$ siècle, dans les textes de Gautier de Coincy (1177/1178-1236), dans les dernières années du $X^{\mathrm{e}}$ siècle, et au début du siècle suivant, avec Eustache Deschamps (1346-1406/1407), l'équivalence est devenue celle des champs de la musique et de la poétique; elle est affichée, comme donnée à voir, à travers la succession des métaphores empruntées à l'une ou à l'autre, et mises au service de l'une et de l'autre ou, plus directement, dans la série des jeux verbaux sur le son comme mot et, inversement, venant achever ainsi une tradition bien plus ancienne, ainsi que l'étudiait Jean-Marie Fritz. L'affirmation disciplinaire est sous-jacente à la base de ces comparaisons en séries et ouvre à la position d'un statut social, puis à sa reconnaissance pour le musicien comme pour les autres «artistes» médiévaux: Vasco Zara a, de la sorte, bien démontré comment, à l'intérieur de ce champ des pratiques et des usages, le «maître ès pierres», plus tard l'architecte, et l'homme de la musique, le «musicien», concevaient, sur des bases intellectuelles très cohérentes, leurs projets et comment ils les faisaient voir: ceci devait conduire à une perception renouvelée de l'homme qui joue d'un instrument de musique, ou qui en joue, mais aussi de l'homme qui mime la musique, le jongleur, ainsi que les travaux de Martine Clouzot l'ont bien fait comprendre ${ }^{3}$.

\section{$2^{e}$ ligne}

5 Dans ce champ tout en tensions, au carrefour de pratiques et d'usages multiples, un outillage mental a été élaboré, puis appliqué, y compris à l'extérieur, vers d'autres disciplines et d'autres savoirs. Je remarquerai que deux principales forces se sont exercées l'une à l'encontre de l'autre, certes, mais aussi l'une à côté de l'autre, en déterminant deux configurations sécantes. 
6 La première correspondit à une formalisation très forte des codes de l'expression du social à travers les registres de ses écritures, mais sans liens très appuyés avec la construction politique d'un État, au moins jusqu'aux années $1150-1200^{4}$. Au cours de nos repérages, nous avons souvent renvoyé aux problématiques abordées par Jack Goody, dans les domaines de l'anthropologie de la connaissance et de ses pratiques ${ }^{5}$, et à celles développées par Mary Carruthers dans son enquête sur le livre de la mémoire ${ }^{6}$, en parlant souvent de mémoire inter-culturelle, entrelacée, toujours plurielle. Dans l'écriture des neumes, par exemple, à propos du manuscrit 44 d'Albi, Eduardo Aubert notait le mélange des raisons internes et externes aux pratiques musicales et leurs conceptions intellectuelles, qui pouvaient rendre compte de l'impossibilité à imposer des normes précises, tandis que Daniel Saulnier remontait à la naissance de ce type d'écriture. Eduardo Aubert examinait encore comment la matérialité multiple du document de base empêchait tout codage et entraînait la nécessaire «mise en registres» (Jack Goody) successifs, qui intégrait des lectures et des modes de déchiffrage multiples, inter-reliés entre eux, en nous faisant pénétrer de la sorte dans la construction intellectuelle et factuelle des neumes. L'étude d'un manuscrit «palimpseste», au sens où Gérard Genette l'entendait, dans toute l'acception (large) du terme ${ }^{7}$, permettait à Christelle Cazaux-Kowalski d'insister, à partir de l'exemple du manuscrit grec 2631 (PARIS, BnF) sur la choséité des feuillets, sur leur présence en tant qu'être-en-soi, résultant d'un montage de séquences autonomes entre elles et ayant, en fin de compte, leur propre devenir, défiant toute tentative à la normalisation.

7 Les impossibilités à normer ne viennent pas seulement des codages et des mises en registres des signes graphiques dans les manuscrits, c'est-à-dire de la «raison graphique» (Jack Goody). Elles tiennent également aux contraintes inhérentes aux genres littéraires et aux statuts qu'on leur attribue: retrouvant le voyage légendaire d'Hucbald à Nevers (vers 870), Jean-François Goudesenne accentuait, avec raison, les implications sur le manuscrit des traditions propres à la philologie, en voie de constitution, et au genre hagiographique, alors en plein essor. Les règles, les prescriptions à suivre éloignaient tout autant que les raisons graphiques du manuscrit de la possibilité d'imposer des normes durables. Enfin, dans la séquence des vingtquatre lettrines illustrées du manuscrit 1 de Semur, à travers la diversité matérielle de leur présence sous la forme de petits «tableaux à part», Daniel Russo voyait à l'œuvre le refus de tout codage externe aux pratiques de l'enlumineur et, aussi, une très forte distance prise par rapport à l'histoire: car, d'une lettrine à l'autre, sur le fond de la trame colorée qu'elles instaurent, à travers la variété qu'elles montrent en action, c'est de mouvement tout orienté vers le devenir, au sens de Gilles Deleuze, qu'il faut parler. C'est dans la dynamique que sont situées leur articulation propre, puis celle qu'elles entretiennent avec le corps du texte, tout aussi mouvant; c'est aussi de cette rencontre que surgit leur statut d'image-présence au sens ontologique de l'expression ${ }^{8}$.

\section{$3^{e}$ ligne}

8 La seconde configuration, sécante de la précédente, repose, au contraire, sur la mise aux normes, en rapports de mieux en mieux pensés avec les catégories qui régissent le gouvernement des hommes en société. Dans les années 1200, et au cours de la première moitié du XIII siècle, à Chartres, à Laon, à Paris, l'équivalence entre musique et rhétorique sert à établir une unité très dense, intellectuelle, sociale et 
politique dans la pensée de l'organum qui va informer les différents aspects des activités humaines 9: Guillaume Gross le reconnaissait dans tout un "parcours de consonances» et dans la manière d' «orner une mélodie sur un mode» ${ }^{10}$; Edgar de Bruyne l'avait aussi analysé comme le mouvement de fond des $\mathrm{XII}^{\mathrm{e}}$-XIII ${ }^{\mathrm{e}}$ siècles, dont la structure est articulée pour être vue sur le mode analogique de l'organisme ${ }^{11}$. À l'autre bout de la chronologie du Moyen Âge, entre la fin du XIV et le début du $\mathrm{Xv}^{\mathrm{e}}$ siècle, dans un champ de métaphores régi par le pouvoir royal, en France par exemple, la mise en forme de la discipline musicale sert d'étalon, selon Jean-Marie Fritz, à tout le champ des pratiques poétiques, celles-ci substituées à la rhétorique ${ }^{12}$. $\mathrm{Et}$, dans la lecture que donnait Grantley McDonald des relectures pour le patriciat de Breslau, à la fin du $\mathrm{Xv}^{\mathrm{e}}$ siècle et au début du siècle suivant, du De passione dominica officium (1516) par Laurentius Corvinus, il devenait évident que la philosophie, et plutôt la philosophie mystique d'inspiration ficinienne, s'inscrivait en lieu et place de la poésie dans le champ de la politique de la ville, comme ceci avait été aussi le cas sous le principat de Laurent le Magnifique à Florence, et sous son immédiat successeur, ainsi qu'André Chastel l'avait bien étudié ${ }^{13}$.

9 Il est d'autres circonstances où l'imposition des normes s'effectue brutalement et sans concessions: par exemple, en situation de polémique, dans un espace tout en conflits, comme on le voit sur le bi-feuillet étudié par Gisèle Clément-Dumas et Isabelle Fabre, dans le manuscrit réalisé à Paris et conservé par la Bibliothèque municipale de Montpellier (fonds Bouhier), en période de polémique anti-judaïque. En relation avec le motet simplex in essentia ${ }^{14}$, avec le texte et avec l'iconographie profane distribuée sur les marges, dans le registre inférieur, une composition unitaire est présentée tel un argument soutenu en quatre points explicites: le son, la voix, l'image, le texte - les quatre points interférant entre eux et affichant, à travers leur diversité, une séquence unitaire empreinte des débats universitaires du moment (vers 1270-1280). Sur ce genre particulier de polémique par l'image, devenue l'alliée de la voix qui chante et qui lit, certains des travaux de Michael Camille sont tout à fait pertinents pour les questions abordées ici ${ }^{15}$.

10 La liturgisation des usages et des contenus est, sans doute, la dernière forme de la remise aux normes, la plus explicite peut-être pour la consonance de la vue et de l'ouïe, la moins perceptible aussi aujourd'hui en raison de l'éloignement dans le temps et des incompréhensions qui en résultent pour les sources. Toutefois, et en dépit de l'absence, qu'il faudra combler, d'un terrain de comparaison avec le monde byzantin chrétien ${ }^{16}$, des pistes très intéressantes ont été déjà largement ouvertes:

11 - sur les rapports entre la mélodie et le sacramentel, avec tous les riches développements qu'en a donnés Nils Holger Petersen;

12 • sur les modes intégrés de fonctionnement entre la liturgie, les hymnes chantés et les peintures, à l'intérieur d'une chapelle bâtie et décorée pour le culte marial, à SaintJulien du Mans, ainsi qu'Helen Deeming l'a noté;

13 • ou encore, sur les transformations en séries opérées, au sein du public de la riche oligarchie de Breslau, par l'interprétation savante de l'office De passione dominica de saint Bonaventure par Laurentius Corvinus (1516), comme l'a démontré Grantley McDonald.

14 Dans toutes ces occurrences, la mise en ordre interfère avec ce qui relève de l'espace et du temps de la musique, je serais tenté de dire «des musiques» : celle des femmes et des 
hommes; celle des princes et des bourgeois des villes; celle des clercs et des laïcs; celle de la terre comme celle des cieux, des catégories que nous devrons nous efforcer, par la suite, de préciser, selon un double intérêt, d'anthropologie sociale et d'histoire, dans la mesure du possible.

\section{$4^{\mathrm{e}}$ ligne}

15 Je soulignerai, en dernière ligne de lecture, et très brièvement, que tous les échanges au cours de ces journées ont fait valoir, de l'analyse à la conceptualisation, la portée des différentes approches du support manuscrit, quel que soit son statut, son genre, son contexte d'insertion sociale et politique, tous ces points sur lesquels il nous faudra aussi revenir à partir du manuscrit envisagé dans sa matérialité ${ }^{17}$. Je remarquerai, du reste, que, toujours, c'est dans la globalité de l'objet posé et dans l'interdisciplinarité des approches en sciences humaines et sociales, que ces résultats stimulants pour la voix, l'œil, et l'esprit, comme le dirait Paul Claudel, ont été atteints. C'est, désormais, à partir de ces résultats qu'il nous faudra reprendre les interrogations pour un approfondissement de nos directions de travail.

\section{Rappel du programme}

L'anachronisme de la notion de «musique médiévale»: outil de recherche ou fantaisie? / The anachronism of the idea of «medieval music»: research tool or fantasy?

-J.-C. schmitT, Rythmus, musica, cantus

- E. øSTREM, Why can't we just assume there is «music» in the Middle Ages (and get on with our work)?

Musique et image: figuration, présentation, représentation / Music and image: figuration, presentation, representation

- D. RUSSO, Séquences figuratives, périmètre visuel, présence ontique dans les arts, $X^{e}-\mathrm{XI}$ siècles

- M.-T. GOUSSET, La représentation des instruments de musique dans l'Apocalypse de 1313 (PARIS, BnF, français 13096)

- M. CLOUZoT, Le jongleur des psautiers, 1220-1390: une inventio musicale et sensorielle retrouvée?

-V.ZARA, Componere ad quadratum: métaphores littéraires et stratégies de composition entre musique et architecture au Moyen Âge

Musique et écriture: annotation, notation, livre / Music and writing: note-taking, notation, book

- D.SAULNIER, Quelques réflexions sur la naissance des écritures neumatiques

-E. HENRIK AUBERT, Les raisons de l'écriture: les logiques plurielles des neumes dans le GraduelAntiphonaire d'Albi (ALBI, BM, 44)

- C. CAZAUX-KOWALSKI, Concevoir un manuscrit musical autour de l'an Mil: un exemple atypique, le Palimpseste de Turin (PARIS, BnF, grec 2631)

- H. DEEMING, La musique silencieuse, le temps statique: l'écriture musicale dans des images au Moyen Âge 
19 Musique et langue: grammaire, rhétorique, chant / Music and language: grammar, rhetoric, chant

-J.-F. GOUDESENNE, Convergences et divergences entre historiographie et philologie: le voyage légendaire d'Hucbald à Nevers (vers 870)

- M. FORMARIER, Le rythme dans les Benedictiones bénéventaines et milanaises. Étude comparative

- D.vAn BETTERAY, Ut secundum sensum verborum cantus varietur: Gregorian chant and its earliest assimilations - a kind of music created by the words and their patristic exegesis and assimilated by reason of pastoral-theological requirements

- G. GROSS, L'organum, une nouvelle parole au treizième siècle?

- G. CLÉMENT-DUMAS et I. FABRE, Le motet comme espace polémique universitaire: l'exemple de Conditio/O natio/Mane prima sabbati du manuscrit de Montpellier

Musique et métaphore: sensibilité, conceptualisation, expression / Music and metaphor: sensibility, conceptualization, expression

- N. H. PETERSEN, The medieval notion of sweet melody

-J.-M. FRITZ, Les métaphores musicales dans la poésie française de la fin du Moyen Âge: traditions, inventions, innovations

- G. MCDONALD, Laurentius Corvinus and the transformation of Bonaventure's De passione dominica officium (1516)

Discussion générale et conclusion / General discussion and conclusion.

\section{NOTES}

1. Sur ce processus bien engagé depuis le XII siècle, cf. J. F. HAMBURGER et A.-M. Bouché (dir.), The Mind's Eye. Art and Argument in the Middle Ages, Princeton U. P., 2006.

2. E. DE BRUYNE, Études d'esthétique médiévale, Bruges, 1946 [rééd. Paris, 1998]; E. PANOFSKY, Gothic Architecture and Scholasticism: An Inquiry into the Analogy of the Arts, Philosophy and Religion in the Middle Ages, Princeton U. P., 1951 [rééd. New York, 1957 ; trad. française et postface P. BouRDIEU, Architecture gothique et pensée scolastique, Paris, 1967, en part. p. 135-167].

3. M. CLouzot, Images de musiciens (1350-1500), Tours/Turnhout, 2008.

4. Pour un exposé d'ensemble, cf. D. BOUTET, Formes littéraires et conscience historique. Aux origines de la littérature française, 1100-1250, Paris, 1999.

5. Voir ses remarques générales dans The Power of the Written Tradition, Washington/Londres, 2000 ; trad. fr. C. MANIEZ, Pouvoirs et savoirs de l'écrit, Paris, 2007; D. RUSSO, "Anthropologie et iconologie : réflexions sur les apports de Jack Goody à la notion de "représentation" ", article en ligne : http://cem.revues.org/document4242.html.

6. M. CARRUTHERS, The Book of Memory. A Study of Memory in Medieval Culture, Cambridge U. P., 1990 ; trad. fr. D. MEUR, Paris, 2002.

7. C'est-à-dire la notion de "réactivation générique» saisie à l'intérieur de la série des transformations successives qui agissent sur un manuscrit, cf. G. GENETTE, Palimpsestes. La littérature au second degré, Paris, 1982, en part. p. 233-236.

8. G. DELEUZE, Cinéma 1. Image-mouvement, Paris, 1983 ; ID., Cinéma 2. Image-temps, Paris, 1985. 
9. Pour un autre champ, qui n'est pas sans liens avec celui que nous avons délimité, voir la synthèse de J. W. BALDWIN, Paris, 1200, trad. française B. BONNE, Paris, 2006.

10. G. GRoss, Chanter en polyphonie à Notre-Dame de Paris aux $12^{e}$ et $13^{e}$ siècles, Turnhout, 2007 (Studia Artistarum, 14), chap. v, VI et VII, en part. p. 215-236.

11. E. DE BRUYNE, Wat is cultuur?, Utrecht, 1937 ; idée reprise et argumentée dans Het Aesthetisch Beleven, Anvers, 1942; sous une forme plus simplifiée dans L'esthétique du Moyen Âge, Louvain, 1947 [rééd. Paris, 1998, t. 2, p. 371-626].

12. J. BLANCHARD et J.-C. MÜHLETHALER, Écriture et pouvoir à l'aube des temps modernes, Paris, 2002.

13. A. CASTEL, Marsile Ficin et l'art, Genève, 1954 ; ID., Art et humanisme à Florence au temps de Laurent le Magnifique. Études sur la Renaissance et l'humanisme platonicien, Paris, 1959.

14. Voir les deux initiales figurées sur le haut de chacun des feuillets, où l'iconographe a choisi deux images trinitaires de Dieu.

15. En particulier M. CAMILLE, The Gothic Idol : Ideology and Making Image in Medieval Art, Cambridge U. P., 1989 et ID., Image on the Edge: The Margins of Medieval Art, New York, 1992 [trad. française B. et J.-C. BONNE, Paris, 1997].

16. En guise d'introduction, voir N. LossKY, Essai sur une théologie de la musique liturgique. Perspective orthodoxe, Paris, 2003.

17. C. DE HAMEL, Une histoire des manuscrits enluminés, Paris, 1995, sur ces « catégorisations » et les façons de les dépasser; en part. p. 9-13.

\section{INDEX}

Mots-clés : musique 